\title{
Medinfo jetzt ausschliesslich online
}

\section{Bruno Soltermann}

Chefarzt Schweizerischer Versicherungsverband und Redaktionseiter des Medinfo
Korrespondenz:

Dr. med. Bruno Soltermann C. F. Meyer-Strasse 14

CH-8022 Zürich

Tel. 0442082828

bruno.soltermann[at]svv.ch
Das Medinfo - Mitteilungen zu Themen der Privatversicherer - wurde letztmals im Dezember 2013 als Beilage zur Schweizerischen Ärztezeitung zugestellt. Es wird weiterhin zweimal im Jahr erscheinen, neu wird es jedoch nur noch elektronisch auf der Webseite des Schweizerischen Versicherungsverbandes einsehbar sein.

Mit der aktuellen Ausgabe des Medinfo möchten wir anstehende Probleme der Transplantationsmedizin aus gesellschaftlicher, ethischer, medizinischer und versicherungsmedizinischer Sicht beleuchten.

Die Resultate in Bezug auf Operationen und Nachsorge sind sehr gut. Die Spendewilligkeit in der Schweiz ist aber im Vergleich zu andern europäischen Ländern klar tiefer. Die ethischen Probleme mit der Akzeptanz von Todeskriterien oder der Allokation wie auch der Umstand, dass durch die Immunsuppression die Morbidität durch Infektionen und Tumoren steigt und die Lebensdauer sinkt, geben zu vielen Diskussionen Anlass. Dieser Umstand schränkt auch die Versicherbarkeit im Zusatzversicherungsbereich ein, was jedoch aufgrund des sehr gut ausgebauten Sozialversicherungsnetzes in der Schweiz für Personen mit Transplantationen kaum eine Rolle spielt.

Die erhöhte Morbidität wird bei Transplantationen von lebenswichtigen Organen hingenommen,

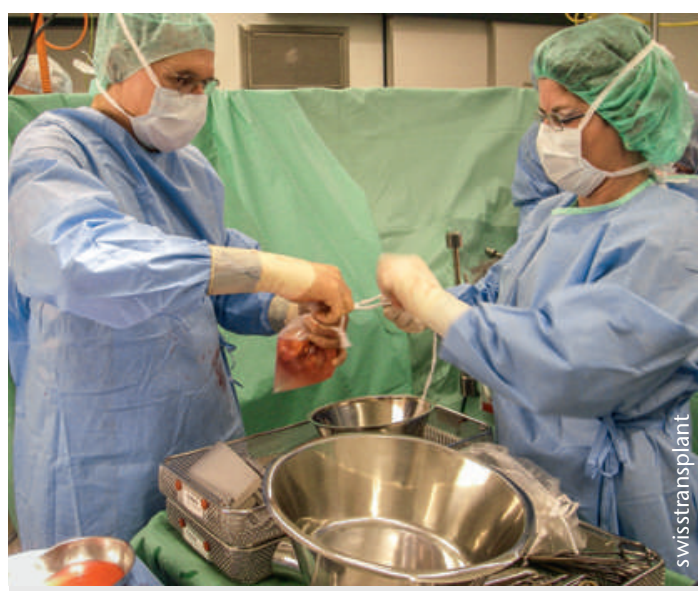

Die Organspende ist Thema der neusten Ausgabe von Medinfo. hingegen bei einer nicht überlebensnotwendigen Handtransplantation nicht, was in der ablehnenden Haltung zur Kostengutsprache für eine Handtransplantation durch die Unfallversicherer führte. In der ablehnenden Begründung wurde zudem darauf hingewiesen, dass es sehr gute Prothesen auf dem Markt gibt. Diese wie auch die äusserst hilfreichen und vielfältigen Orthesen werden kurz und lehrreich mit ihren Indikationen sowie den Kosten und dem Nutzen dargestellt.

Das Medinfo können Sie unter www.svv.ch/de/ publikationen/medinfo-mitteilungen-zu-themender-privatversicherer einsehen.

\section{Inhaltsverzeichnis Medinfo \\ Franz F. Immer: \\ Organspende und Transplantationen in der Schweiz: Quo Vadis?}

\section{Markus Zimmermann:}

Ethische Überlegungen zu einigen Herausforderungen der Transplantationsmedizin

Beat Kipfer:

Versicherbarkeit nach Transplantationen

Reinald Brunner:

Kosten und Nutzen von Orthesen

Thomas Böni, Bruno Soltermann:

Kosten und Nutzen orthopädischer Prothesen

F. Rota, J.-D. Sauvant, R. Marelli, O. Ducarre,

T. Lack, B. Soltermann:

Zusammenarbeit zwischen Fachärzten für Psychiatrie und Psychotherapie und Case Managern der Privatversicherer / Coopération entre médecins spécialistes en psychiatrie et psychothérapie et case managers des assureurs privés / Collaborazione tra medici specialisti in psichiatria e psicoterapia e case manager degli assicuratori privati 